\title{
A televideo exercise and nutrition program for children with acute lymphoblastic leukemia in maintenance therapy: design and methods
}

This article was published in the following Dove Press journal:

Open Access Journal of Clinical Trials

29 July 2015

Number of times this article has been viewed

\author{
Cheryl A Gibson' \\ Keith J August ${ }^{2}$ \\ Jerry L Greene ${ }^{3}$ \\ Stephen D Herrmann ${ }^{4}$ \\ Jaehoon Lee ${ }^{5}$ \\ Susan P Harvey ${ }^{6}$ \\ Kate Lambourne ${ }^{3}$ \\ Debra K Sullivan ${ }^{7}$ \\ 'Department of Internal Medicine, \\ Division of General and Geriatric \\ Medicine, University of Kansas \\ Medical Center, KS, USA; ${ }^{2}$ Children's \\ Mercy Hospital, MO, USA; \\ ${ }^{3}$ Department of Health, Sport, \\ and Exercise Sciences, University \\ of Kansas, KS, USA; ${ }^{4}$ Children's \\ Health Research Center, Sanford \\ Research, SD, USA; Institute for \\ Measurement, Methodology, Analysis \\ and Policy, Texas Tech University, \\ TX, USA; ${ }^{6}$ Center for Research on \\ Learning, University of Kansas, KS, \\ USA; ${ }^{7}$ Department of Dietetics and \\ Nutrition, University of Kansas \\ Medical Center, KS, USA
}

Correspondence: Cheryl A Gibson Department of Internal Medicine, University of Kansas Medical Center, 390I Rainbow Boulevard, MSI020,

Kansas City, KS 66160, USA

Tel + I 9135887207

Fax + I 9135883877

Email cgibson@kumc.edu
Abstract: Changes in nutrient intake and decreased exercise resulting from cancer therapies as well as their side effects may be contributing factors in the increased body weight and differences in physical fitness observed in survivors of childhood acute lymphoblastic leukemia (ALL). This article will describe the study protocol for an intervention program designed to improve the physical activity and nutrition behaviors of ALL survivors. Twenty-four children aged between 4 years and 12 years with ALL will be randomized to a 6-month technology-based exercise and nutrition program (TLC4ALLKids) or to enhanced usual care (eUC). The participants randomized to the TLC4ALLKids will participate in weekly, 1-hour coaching sessions on nutrition and physical activity and 1-hour physical activity classes delivered by group video conferencing. Participants will be provided with iPad tablets loaded with video conferencing software and the Healthy Lifestyle Tracking calendar to track daily nutrition and physical activity goals and weight. Both groups will be provided with Fitbit ${ }^{\mathrm{TM}} \mathrm{Zip}$ to monitor physical activity. To assess feasibility, participant recruitment (achievement of proposed sample size), attendance (per weekly online sessions/assessment sessions), and adherence (number of families at 3 and 6 months) will be evaluated. Outcome measures to assess the intervention will include anthropometrics (weight, height, and waist circumference), physical activity (accelerometry), energy and macronutrient intake (food records), sleep habits (Children's Sleep Habits Questionnaire), and quality of life (Pediatric Quality of Life Inventory) will be obtained at baseline, 3 months, and 6 months. Semistructured interviews will be used to gather information about ways to improve the program and overcome barriers to participation. If successful, the TLC4ALLKids intervention will provide a means to educate and improve the health behaviors of ALL survivors that can be delivered remotely and conveniently to participants.

Keywords: acute lymphoblastic leukemia, enhanced usual care, social cognitive theory

\section{Introduction}

Acute lymphoblastic leukemia (ALL) is one of the most common childhood cancers, accounting for $74 \%$ of all leukemia cases in patients aged $0-19$ years in $2010 .{ }^{1}$ Due to improved treatments, survival rates are approaching $90 \%$ for children with ALL. ${ }^{2}$ However, treatment options such as radiation, chemotherapy, and corticosteroids have been associated with growth hormone deficiency, central obesity, hyperlipidemia, and other components of cardiovascular disease and metabolic syndrome. ${ }^{3,4}$ Poor dietary habits and physical inactivity also contribute to excessive weight gain following ALL treatment. Studies evaluating nutrient intake of adult survivors of childhood ALL have shown excessive consumption of sodium, refined sugar, and meat ${ }^{5}$ and too few fruits, vegetables, whole grains, and calcium. ${ }^{6}$ Furthermore, research indicates 
that survivors of childhood cancers are less likely to be active than non-cancer survivors, ${ }^{7}$ which may be a result of chemotherapy-induced neuropathy and muscle atrophies. ${ }^{8,9}$ Helping survivors of ALL establish healthier eating patterns and more active lifestyles is essential to their prolonged survival and quality of life.

Home-based programs are a convenient way to deliver exercise and nutrition interventions to young ALL survivors because participants are not required to secure travel arrangements or risk potential infection. Utilizing technologies such as group video conferencing, Fitbit ${ }^{\mathrm{TM}} \mathrm{Zip}$, and accelerometers allows interventionists to deliver programs to a group of individuals and track physical activity (PA) behaviors remotely. When reinforced by follow-up phone calls and health provider counseling, home-based exercise programs have been shown to improve fitness, body composition, physical functioning, and decrease fatigue in adolescents and adults. ${ }^{10-12}$ Researchers have evaluated an individualized home-based exercise and nutrition program in response to the need for improvements in nutrition and PA for 4-year-old to 10-year-old survivors of ALL, comparing habitual lifestyle exercise and nutritional intake patterns in ALL survivors compared to controls. ${ }^{13} \mathrm{PA}$ as measured by fitness tests, self-report, and pedometer steps increased in the treatment group compared to the control group. However, the nutrient and energy intakes of treatment and control groups did not differ. Additional approaches are needed to alter the nutrient intakes of this population and establish the best family-based strategies to do so. This study will utilize technology to deliver an exercise and nutrition program remotely to a group of ALL survivors with weekly counseling sessions to teach participants and their families about nutrition, PA, and behavioral strategies tailored to families coping with cancer. The use of telemedicine has been growing and increasingly applied to different areas of medical practice and the application to health behaviors of pediatric cancer survivors could increase the reach of efficacious interventions. ${ }^{14}$

This article will outline the study protocol for a feasibility study of an intervention program designed for 4-year-old to 12-year-old ALL survivors and their families. Participants will be randomized to either a technology-based lifestyle modification program (TLC4ALLKids) or to enhanced usual care (eUC). Outcome variables will include participant recruitment (achievement of proposed sample size), attendance (per weekly online sessions/assessment sessions), and adherence (number of families at 3 months and 6 months). A secondary purpose of the feasibility study will be to estimate the effectiveness of the intervention program in producing changes in PA, fruit and vegetable intake, low-fat dairy consumption, water consumption, and changes in BMI (body mass index) appropriate for growth and development at 3 and 6 months.

\section{Materials and methods Overview of study design}

The proposed feasibility study will draw from the social cognitive theory (SCT) of behavioral change. ${ }^{15}$ The SCT explains behavior as a triadic relationship between personal, behavioral, and environmental factors. The particular constructs targeted and operationalized in this intervention include self-efficacy, outcome expectations, self-monitoring, goal setting, perceived facilitators and barriers to changes, role modeling, and environmental factors. All of these constructs have been shown to be related to children's fruit and vegetable consumption ${ }^{16-20}$ and enhanced PA levels. ${ }^{21,22}$ The study will utilize a randomized controlled trial design in which 24 children with ALL be randomized either to a technology-based lifestyle modification program (TLC4ALLKids) or to eUC.

\section{Participant eligibility}

ALL therapy consists of a 6-9 month intensive treatment period following diagnosis, then a less intense maintenance treatment that lasts for 2-3 years. Maintenance treatment involves clinic visits every 4 weeks and includes pulse dosing of glucocorticoids (dexamethasone or prednisone). The maintenance phase is being targeted for this intervention because this is the time when children are at the greatest risk for gaining weight, and physicians and healthcare teams begin to focus helping families resume normal routines and activities that promote health maintenance.

The inclusion criteria for participation in this study include: 1) families of children aged 4-12 years with ALL who have entered the maintenance phase of treatment for at least 4 months but not longer than 12 months; 2) availability to participate in assessments over 6 months; 3 ) parent and child having the ability to speak and understand English; 4) ability to report data weekly by at least one of three alternative methods: telephone, email, or fax; and 5) access to wireless Internet (cable or DSL connection). Participants will be excluded from the study due to: 1) participation in another childhood weight management, nutrition, or PA program; 2) diagnosis of a psychiatric disease such as depression, bipolar disorder, or autism spectrum disorder; 3) dietary restrictions, such as vegetarianism or severe food allergies; 4) inability to perform moderate 
to vigorous PA; 5) unwillingness to be randomized, and 6) receiving supplemental nutrition (ie, total parenteral nutrition, nasogastric tube feedings, etc).

\section{Recruitment and randomization}

Families of children treated for ALL who are in the maintenance phase will be recruited from the Pediatric Oncology Clinic of Children's Mercy Hospital (CMH) in Kansas City, Missouri. Potential families will be invited to participate in the study at their monthly visit with the oncologist during the maintenance phase of ALL treatment. Interested families will be screened for eligibility by the study coordinator via telephone call after the oncologist has invited families to participate. Informed consent from a parent and child assent will be obtained at an informational meeting held prior to enrollment. This study was approved by the Human Subjects Committee at the University of Kansas Medical Center and the Protocol Review and Monitoring Committee (PRMC) of the University of Kansas Cancer Center.

To reduce travel costs, families in both groups will be scheduled for baseline assessments during monthly pediatric oncology visits, during which sociodemographic information and medication history will be collected. Sociodemographic variables will include ethnicity, race, sex, age, and parent education and marital status at baseline. The patient's medications as well as treatment plans throughout the 6-month study will also be assessed. This is to document and account for when participants complete assessments while on steroids since the delivery of steroid pulses to patients could have a profound impact on the study parameters. This issue will be addressed by tracking the dates of diet/activity records. Finally, anthropometric measures and questionnaires to assess sleep and quality of life will be collected during the baseline visit, and assessments will take approximately 15 minutes to complete.

Prior to assessments, participants will be randomized using a table of random numbers so that half of the enrolled children will be assigned to receive the TLC4ALLKids, and the other half will be assigned to receive the eUC with an equal probability of the assignments. Randomization will be completed by the study statistician who will not be involved in the intervention delivery or data collection.

\section{Intervention}

\section{Lifestyle modification group (TLC4ALLKids)}

Families randomized to the TLC4ALLKids will participate in weekly, 1-hour health coaching sessions delivered by video conferencing on the iPad tablet utilizing the GoToMeeting ${ }^{\mathrm{TM}}$
(Citrix Online LLC, Santa Barbara, CA, USA) platform. GoToMeeting ${ }^{\mathrm{TM}}$ will allow the health educator to conduct group health coaching sessions in which all group members can see one another. Children in the maintenance phase of treatment for ALL may be at risk for potential infections and may be reluctant to attend in-person sessions. The video conferencing method allows the child to be a part of a group, converse with peers and receive peer support, and avoid barriers such as travel costs, lack of transportation, etc. GoToMeeting ${ }^{\mathrm{TM}}$ sessions will be conducted via the iPad, and the system includes the unique feature of allowing the health educator to present documents live on screen and to highlight aspects of the presentation or topic.

These sessions will teach families about nutrition, PA, and behavioral strategies tailored to families dealing with cancer (Table 1 for topic outlines). They will also be taught healthy eating goals for children using the Dietary Guidelines and MyPlate goals. Additionally, families will be taught appropriate portion sizes and healthy cooking skills, and will receive a cookbook titled, "Happily Hungry: Smart Recipes for Kids with Cancer." Specific issues related to cancer survivors such as exercise capacity, nutrient needs, and parenting habits will be addressed. The TLC4ALLKids group will participate in weekly 1-hour health coaching sessions delivered by video conferencing. The first 15-20 minutes of the session will include problem solving between children and the health coach as well as interactive activities focusing on nutrition, PA, and behavior change. The remaining 40-45 minutes will

Table I Sample topics and goals for TLC4ALLKids intervention

\begin{tabular}{|c|c|}
\hline Wee & ioals \\
\hline $\begin{array}{l}\text { I. Program introduction } \\
\text { Physical Activity Guidelines Nutrition } \\
\text { Guidelines and Daily Tracking } \\
\text { Physical activity: Beginning } \\
\text { Exercises }\end{array}$ & $\begin{array}{l}\text { Nutrition: } 3 \text { F/V, } 2 \text { LFD, } 2 \text { W } \\
\text { PA: } 2 \text { days for } 20 \text { minutes each } \\
\text { Steps: Get a baseline number } \\
\text { of steps }\end{array}$ \\
\hline $\begin{array}{l}\text { Fitness for survivors } \\
\text { FITT Principles and Safety Tips } \\
\text { Flexibility and Resistance Training } \\
\text { Physical activity: Tennis Ball } \\
\text { Fitness and Fun }\end{array}$ & $\begin{array}{l}\text { Nutrition: } 3 \text { F/V, } 2 \text { LFD, } 2 \text { W } \\
\text { PA: } 2 \text { days for } 25 \text { minutes each } \\
\text { Steps: Increase number of } \\
\text { steps by } 500 \text { daily }\end{array}$ \\
\hline $\begin{array}{l}\text { Nutrition: goal setting } \\
\text { Parental and Peer Role Modeling } \\
\text { Rewarding Accomplishments } \\
\text { Physical activity: Magic Rope } \\
\text { Jumping }\end{array}$ & $\begin{array}{l}\text { Nutrition: } 4 F / V, 3 \text { LFD, } 3 \mathrm{~W} \\
\text { PA: } 3 \text { days for } 25 \text { minutes each } \\
\text { Steps: Increase number of } \\
\text { steps by } 500 \text { daily }\end{array}$ \\
\hline $\begin{array}{l}\text { 4. Nutrition: managing mealtime } \\
\text { Making MyPlate Work for You } \\
\text { Meal Planning/Grocery Shopping } \\
\text { Physical activity: Throw and Go }\end{array}$ & $\begin{array}{l}\text { Nutrition: } 4 \text { F/V, } 3 \text { LFD, } 3 \mathrm{~W} \\
\text { PA: } 3 \text { days for } 30 \text { minutes each } \\
\text { Steps: Increase number of } \\
\text { steps by } 500 \text { daily }\end{array}$ \\
\hline
\end{tabular}

Abbreviations: F/V, fruits and vegetables; LFD, low-fat dairy; W, water; PA, physical activity; FITT, Frequency, Intensity, Time, Type. 
consist of discussion between the health coach and parents about how to encourage active play, incorporate healthy foods into the family meals, and promote healthy behaviors. Each iPad will be equipped with the bookcase feature whereby weekly lessons can be stored in PDF form. Attendance will be taken at each meeting. Finally, the GoToMeeting ${ }^{\text {TM }}$ platform will allow the study team to record sessions to be transcribed verbatim for quality and uniformity purposes.

Children will also participate in weekly 1-hour classes led by an adaptive PA expert and delivered by video conferencing. These sessions are designed to provide education about PA opportunities for the children. They will also promote PA with a goal of three 10-15 minute bouts of moderate intensity PA (ie, 3-6 metabolic equivalent of tasks [METs]) performed daily in the home setting. Parents will be instructed to encourage their children to accumulate at least 150 minutes of moderate intensity PA per week, as tolerated. Research results indicate interventions should focus on family and peer support to increase child survivors' PA behaviors. ${ }^{22}$ This approach also satisfies the American College of Sports Medicine, Centers for Disease Control and Healthy People 2010 Guidelines for PA, and provides PA to children in a context other than physical education or competitive sports. Further, with this technology, the children will be able to see and interact with each other during the PA sessions without direct physical contact.

Given the course of treatment, its effects on motor skills and physical capacity, and the importance of injury prevention, the PA program will progress slowly and move to a moderate intensity level over a period of several weeks. Further, an adaptive PE specialist who has extensive experience with working with children with chronic illnesses was selected to design the PA program. During the first session, the specialist will teach families to monitor the perceived exertion of their child during PA, using the Borg's Rate of Perceived Exertion scale. ${ }^{23}$ Based on how their body feels, the participants will be instructed to increase or decrease their efforts accordingly.

\section{eUC group}

Families randomized to the eUC group will be provided standard recommendations from the pediatric oncologist to eat a well-balanced diet and to perform PA as tolerated. In addition, families in the eUC group will be provided iPads and Fitbit $^{\text {TM }}$ Zips but will not attend weekly health coaching or PA classes, using iPad technology. The iPads will contain the same applications (ie, Fitbit ${ }^{\text {TM }} \mathrm{Zip}$ ) as the intervention group to collect nutrition and PA data during assessment periods. Educational materials shared with families at the clinic by medical staff will be made accessible on the iPad as PDFs. This information will be stored using the bookcase feature of the iPad so that families can retrieve these educational materials at any time.

\section{Sources for data collection iPad tablets}

Families will be provided with an Apple iPad tablet to be used during the 6-month study. The iPads will be used to conduct weekly health coaching sessions via video conferencing for the TLC4ALLKids group. Each iPad will be loaded with apps relevant to the study, including GoToMeeting ${ }^{\mathrm{TM}}$, Fitbit ${ }^{\mathrm{TM}} \mathrm{Zip}$, and the Healthy Lifestyle Tracking Calendar used to record targeted healthy behaviors. For the eUC group, information loaded on the iPad will include educational materials shared by medical staff at the clinic and Fitbit ${ }^{\mathrm{TM}} \mathrm{Zip}$. Children in both groups will be provided with a Fitbit ${ }^{\mathrm{TM}} \mathrm{Zip}$ (Fitbit Inc., San Francisco, CA, USA), which is an accelerometer and pedometer in one that wirelessly syncs to the Fitbit ${ }^{\mathrm{TM}} \mathrm{Zip}$ web-based application. The Fitbit ${ }^{\mathrm{TM}} \mathrm{Zip}$ will allow families to track their daily step totals, view graphs of their progress, and keep records. Fitbit ${ }^{\mathrm{TM}} \mathrm{Zip}$ will be provided at the consenting session. Parents will be shown how the Fitbit ${ }^{\mathrm{TM}} \mathrm{Zip}$ should be worn and asked to ensure his/her child wears the pedometer all the time except during bathing, showering, or swimming. Participants in the TLC4ALLkids group will be encouraged to wear Fitbit ${ }^{\mathrm{TM}} \mathrm{Zip}$ for the first 3 months of the study and bi-weekly for the remainder of the study. The participants in the eUC group will be encouraged, but not reminded, to wear the Fitbit ${ }^{\mathrm{TM}} \mathrm{Zip}$ during the 6 months of the study. The number of steps and minutes of PA will be assessed for both groups. Based on a study conducted by Tudor-Locke et al, ${ }^{24}$ a minimum of 12,000 steps per day will be targeted for both sexes by the end of the 6-month intervention for the TLC4ALLKids group. Step goals will be increased over time using a slow progression goal system, as tolerated. The Fitbit ${ }^{\mathrm{TM}} \mathrm{Zip}$ data will be used as a way to provide feedback about the TLC4ALLKids' daily PA and to help set weekly goals. Families in both groups will be allowed to keep the iPad tablet and Fitbit ${ }^{\mathrm{TM}} \mathrm{Zip}$ following participation in the study.

\section{Measures}

Outcome assessments including anthropometrics (weight, height, and waist circumference), PA (accelerometry), and energy and macronutrient intake (food records) for both intervention groups will be obtained at baseline, 3 months, and 6 months by trained research assistants. Given the nature of the intervention, it will not be possible to blind either participants or those administering the interventions to group assignment. 
However, the participants will not be aware of the study hypotheses. Data collection will be completed in a private clinic office for participants in the TLC4ALLKids and eUC groups to avoid any potential contamination among participants.

\section{Anthropometrics}

Height $( \pm 0.1 \mathrm{~cm})$ will be measured, without shoes, using a Seca stadiometer (Model 214, Seca Corporation, Culver City, CA, USA). Body weight $( \pm 0.2 \mathrm{~kg})$, wearing light clothing without shoes, will be measured using a digital scale (TBF310 GS, Tanita Corporation, Tokyo, Japan). BMI $\left(\mathrm{kg} / \mathrm{m}^{2}\right)$ will be calculated. BMI $z$ values ( $z$-BMI) standardized for child age and sex will be calculated based on comparison to population norms. ${ }^{25}$ Waist circumference will be obtained with two measurements per site within $2 \mathrm{~cm}$. The circumference will be measured using the procedures of Lohman et al. ${ }^{26}$ Blood pressure will be obtained from the child's medical chart. During the blood pressure measurement, the child will be seated for a minimum of 5 minutes in an isolated room with the arm bared, supported, and positioned at the heart level. A cuff will be selected based on the measurement of the length and circumference of the arm. Systolic and diastolic pressures will be recorded. Two measures will be averaged and additional measures will be obtained if the measures differ by more than $5 \mathrm{mmHg} .{ }^{27}$ Height and body weight, waist circumference, and blood pressure measurements will be taken at baseline, 3 months, and 6 months.

\section{Physical activity}

Children participating in the study will be asked to wear a PA monitor called an ActiGraph accelerometer (ActiGraph IIc, Pensacola, FL, USA) at baseline, 3 months, and 6 months. Parents will be instructed on how the accelerometer should be worn and asked to help their child place the accelerometer each day. Accelerometers are to be worn at the beginning when the child wakes up and ending when the child goes to bed except for bathing, showering, or swimming. Children will wear accelerometers for 7 consecutive days at each assessment period. Researchers will mail accelerometers to the participants prior to their monthly oncology visits and collect them during these visits.

\section{Dietary assessment}

Three-day food intake records ( 2 week days and 1 weekend day) will be completed for the child by parents of both groups during the first week for baseline values prior to intervention, and at 3 months and 6 months. Parents will be asked to record three-day food intake records as well as provide photographs of these food items. A registered dietitian will review records with parents during the monthly oncology clinic visits. Information gained from the food records will be used to complete the Healthy Eating Index, which is based on the MyPlate food group recommendations. ${ }^{28}$ This index is a way to evaluate the quality of an individual's diet and can be used for all ages. Serving sizes for each food group will be based on the Dietary Guidelines for Americans 2010 (US Department of Agriculture and US Department of Health and Human Services).

\section{Quality of life}

The Pediatric Quality of Life Inventory (PedsQL) is a modular instrument designed to measure health-related quality of life (HRQOL) in children and adolescents aged 2-18 years. ${ }^{29}$ The PedsQL 4.0 Generic Core Scales are multidimensional child self-report and parent proxyreport scales developed as the generic core measure to be integrated with the PedsQL disease-specific modules. The PedsQL Multidimensional Fatigue Scale is designed to measure fatigue in pediatric patients. The PedsQL 3.0 Cancer Module is designed to measure pediatric cancerspecific HRQOL. ${ }^{30}$ These instruments will be administered at baseline and 6 months.

\section{Sleep habits}

The Children's Sleep Habits Questionnaire (CSHQ) is an instrument designed to measure the overall sleep habits in children aged 4-12 years. This instrument will be administered at baseline, 3 months, and 6 months.

\section{Semi-structured interviews}

The study team will invite parents and their child to participate in a semi-structured interview for both groups. The interviewer will inquire about how the program could be improved and solicit information about the benefits and barriers to participation. While telehealth is a promising avenue, it must be accepted by the population to which it is applied to be effective, yet this area is understudied..$^{31}$ For this reason, the interview will include questions about the social acceptability of and confidence in the effectiveness of the telehealth technology. Interviews will be recorded and transcribed verbatim. All data will be entered into a qualitative data software program (Atlas.ti 7, Berlin, Germany) to facilitate the storage, searching, and coding of data. Investigators will explore trends and identify common themes across interviews. 


\section{Process data collection}

Children randomized to the TLC4ALLKids group will track daily nutrition and PA goals as well as their weekly weight using the Healthy Lifestyle Tracking calendar loaded on the iPad. The data collection will include the number of servings of fruits, vegetables, low-fat dairy, and water consumed as well as the number of steps taken and minutes of PA completed daily. Completed Healthy Lifestyle Tracking Calendars will be submitted weekly. Data collected will be utilized to give constructive feedback to the TLC4ALLKids group regarding their progress in increasing nutrition and PA behaviors. For the eUC group, data will be collected at baseline, 3 months, and 6 months, during the participant's monthly clinic visit.

\section{Analyses}

A qualitative analysis on responses from the semi-structured interviews will be performed. Descriptive statistics will summarize participant sociodemographics and all outcomes at each assessment, and bivariate tests (eg, $t$-test, $\chi^{2}$ test, etc) will compare the means and distributions of these variables between and within intervention groups. Mixed linear models will compare outcomes of the TLC4ALLKids and eUC, and study the changes (ie, developmental pattern) in outcomes over a 6-month study period. The sociodemographic variables found to significantly differ between the two groups will be included in the models as potential covariates, thereby providing unbiased estimates of the intervention effects. Because this is a feasibility study, intent-to-treat analyses will be conducted ie, partial sets of outcome measurements are not lost but analyzed in the mixed modeling. ${ }^{32}$ As a secondary intent-to-treat analysis, missing data will be handled by utilizing Markov Chain Monte Carlo multiple imputation technique. ${ }^{32} \mathrm{~A}$ large number (eg, 200) of complete datasets will be generated, and then results from each complete dataset will be combined to make valid statistical inferences..$^{33}$ All the proposed analyses will be performed using SAS 9.4..$^{34}$

\section{Power and sample size}

Sample size was determined to provide sufficient power for the secondary analysis of evaluating the effectiveness of the TLC4ALLKids. Previous studies of home-based exercise and nutrition programs suggest that the present study will achieve large effects - eg, Cohen's $f=0.84$ for PA (steps per day) $;{ }^{13} f=0.59$ and 0.51 for HRQOL and fatigue, respectively; ${ }^{35}$ and $f=0.71$ for daily fruit consumption. ${ }^{18}$ With these effect size estimates, an assumed correlation of 0.30 among repeated assessments, and a Type I error rate of 5\%, a sample of 20 children is expected to provide power of at least $84 \%$. Assuming a conservatively high attrition rate of $15 \%, 12$ children will be randomized to each group in this study (anticipated final $N \geq 20$ children in total). Although power for anthropometric outcomes (ie, BMI) would not reach $80 \%$, overall this study will be adequately powered to address the impacts of the TLC4ALLKids intervention.

\section{Discussion}

Development and evaluation of interventions to improve the health of children after cancer, who are at high risk for lifelong complications, is extremely important. Recent reviews of the literature suggest that childhood cancer survivors engage in health-promoting behaviors at insufficient rates that are similar to those in the general population. ${ }^{7}$ Decreased PA levels among pediatric survivors has been linked to the development of metabolic syndrome, ${ }^{3}$ poor bone mass density and osteoporosis risk, ${ }^{36}$ and higher fat mass. ${ }^{37} \mathrm{~A}$ recent metaanalysis showed that obesity is prevalent in ALL survivors and is independent of patient characteristics and treatment approaches. ${ }^{38}$

Health complications could be minimized through the adoption of healthy behaviors, including nutrition and appropriate types of PA. Current research aims to improve nutrition and PA following intensive treatment to help survivors to achieve or maintain a healthy weight. Most nutrition experts recommend that pediatric cancer survivors eat more fruits, vegetables, and whole grain products, and to lead lifestyles that are more active. Recently, efforts have been made to use home-based delivery of programs to promote healthy eating, PA, and weight control to children survivors of ALL. ${ }^{13}$ However, strategies to enhance the nutritional quality among this population are greatly needed.

If successful, this technology-based intervention (TLC4ALLKids) will provide a convenient way to deliver health information remotely to children who are at risk for infections and could greatly benefit from a home-based intervention. Utilizing technology to connect children to health educators as well as their peers also provides them with important social contact and support.

\section{Acknowledgments}

The study was funded by the St Baldrick's Foundation. We thank Taylor Young, RDN, and Ann Swanson, RDN for their assistance in developing the nutrition topics and thoughtful comments during the design of the study.

\section{Disclosure}

The authors report no conflicts of interest in this work. 


\section{References}

1. American Cancer Society. Cancer Facts and Figures 2010. Atlanta, GA: American Cancer Society, Inc., 2010.

2. Hunger SP, Lu X, Devidas M, et al. Improved survival for children and adolescents with acute lymphoblastic leukemia between 1990 and 2005: a report from the children's oncology group. J Clin Oncol. 2012;30(14):1663-1669

3. Hoffman KE, Derdak J, Bernstein D, et al. Metabolic syndrome traits in long-term survivors in pediatric sarcoma. Pediatr Blood Cancer. 2008;50(2):341-346.

4. Oeffinger KC, Buchanan GR, Eshelman DA, et al. Cardiovascular risk factors in young adult survivors of childhood acute lymphoblastic leukemia. J Pediatr Hematol Oncol. 2001;23(7):424-430.

5. Robien K, Ness KK, Klesges LM, Baker KS, Gurney JG. Poor adherence to dietary guidelines among adult survivors of childhood acute lymphoblastic leukemia. J Pediatr Hematol Oncol. 2008;30(11):815-822.

6. Demark-Wahnefried W, Werner C, Clipp EC, et al. Survivors of childhood cancer and their guardians. Cancer. 2005;103(10):2171-2180.

7. Stolley MR, Restrepo J, Sharp LK. Diet and physical activity in childhood cancer survivors: a review of the literature. Ann Behav Med. 2010;39:232-249.

8. Warner JT, Bell W, Webb DK, Gregory JW. Daily energy expenditure and physical activity in survivors of childhood malignancy. Pediatr Res. 1998;43:607-613.

9. van Brussel M, Takken T, van der Net J, et al. Physical function and fitness in long-term survivors of childhood leukaemia. Pediatr Rehabil. 2006;9(3):267-274

10. Wilson RW, Jacobsen PB, Fields KK. Pilot study of a home-based aerobic exercise program for sedentary cancer survivors treated with hematopoietic stem cell transplantation. Bone Marrow Transplant. 2005;35(7):721-727

11. Burke V, Milligan RA, Thompson C, et al. A controlled trial of health promotion programs in 11-year-olds using physical activity "enrichment" for higher risk children. J Pediatr. 1998;132(5):840-848.

12. Patrick K, Calfas KJ, Norman GJ, et al. Randomized controlled trial of a primary care and home-based intervention for physical activity and nutrition behaviors: PACE+ for adolescents. Arch Pediatr Adolesc Med 2006;160(2):128-136.

13. Moyer-Mileur LJ, Ransdell L, Bruggers CS. Fitness of children with standard-risk acute lymphoblastic leukemia during maintenance therapy: response to a home-based exercise and nutrition program. $J$ Pediatr Hematol Oncol. 2009;31:259-266.

14. Di Cerbo A, Morales-Medina JC, Palmieri B, Iannitti T. Narrative review of telemedicine consultation in medical practice. Patient Prefer Adherence 2015 Jan 13; 9:65-75. Doi: 10.2147/PPA.S61617.)

15. Bandura A. Social Foundations of Thought and Action: a Social Cognitive Theory. New York, NY: Prentice Hall; 1986.

16. De Bourdeaudhuij I, te Velde S, Brug J, et al. Personal, social and environmental predictors of daily fruit and vegetable intake in 11-yearold children in nine European countries. Eur J Clin Nutr. 2007;62(7): 834-841.

17. Cooke LJ, Wardle J, Gibson EL, Sapochnik M, Sheiham A, Lawson M. Demographic, familial and trait predictors of fruit and vegetable consumption by pre-school children. Public Health Nutr. 2004;7(2): 295-302.

18. Pearson N, Biddle SJ, Gorely T. Family correlates of fruit and vegetable consumption in children and adolescents: a systematic review. Public Health Nutr. 2009;12(2):267-283.
19. Resnicow K, Davis-Hearn M, Smith M, et al. Social-cognitive predictors of fruit and vegetable intake in children. Health Psychol. 1997;16(3):272-276.

20. Golan M, Crow S. Parents are key players in the prevention and treatment of weight-related problems. Nutr Rev. 2004;62(1):39-50.

21. Ely AC, Befort C, Banitt A, Gibson C, Sullivan D. A qualitative assessment of weight control among rural Kansas women. J Nutr Educ Behav. 2009;41(3):207-211.

22. Gilliam MB, Madan-Swain A, Whelan K, Tucker DC, DemarkWahnefried W, Schwebel DC. Social, demographic, and medical influences on physical activity in child and adolescent cancer survivors. J Pediatr Psychol. 2012;37(2):198-208.

23. Borg GA. Psychophysical bases of perceived exertion. Med Sci Sports Exerc. 1982;14(5):377-381.

24. Tudor-Locke C, Pangrazi RP, Corbin CB, et al. BMI-referenced standards for recommended pedometer-determined steps/day in children. Prev Med. 2004;38:857-864.

25. Centers for Disease Control and Prevention. Percentile Data Files with LMS Values; 2000. Available from: http://www.cdc.gov/growthcharts/ percentile_data_files.htm. Accessed March 12, 2013.

26. Lohman TG, Roche AF, Martorell R. Anthropometric Standardization Reference Manual. Champaign, Ill: Human Kinetics Books; 1988.

27. Luepker RV, Jacobs DR, Prineas RJ, Sinaiko AR. Secular trends of blood pressure and body size in a multi-ethnic adolescent population: 1986 to 1996. J Pediatr. 1999;134(6):668-674.

28. United States Department of Agriculture. Center for Nutrition Policy and Promotion. USDA, CFNPAP. Healthy Eating Index; 2010. Available from: http://www.cnpp.usda.gov/healthyeatingindex.htm. Accessed March 17, 2011

29. Varni JW, Burwinkle TM, Seid M, Skarr D. The PedsQL 4.0 as a pediatric population health measure: feasibility, reliability, and validity. Ambul Pediatr. 2003;3(6):329-341.

30. Varni JW, Burwinkle TM, Katz ER, Meeske K, Dickinson P. The PedsQL in pediatric cancer: reliability and validity of the pediatric quality of life inventory generic core scales, multidimensional fatigue scale, and cancer module. Cancer. 2002;94(7):2090-2106.

31. Poder TG, Bellemare CA, Bedard SK, Lemieuz R. Social acceptance and population confidence in telehealth in Quebec. BMC Health Serv Res 2015 Feb 21; 15:72.

32. Enders C. Applied Missing Data Analysis. New York, NY: Guilford Press; 2010.

33. Rubin DB. Multiple Imputation for Nonresponse in Surveys. New York, NY: John Wiley \& Sons, Inc.; 1987.

34. SAS Institute. SAS/STAT 9.4 User's Guide. Cary, NC: SAS Institute, Inc.; 2002-2012.

35. Keats MR, Culos-Reed SN. A community-based physical activity program for adolescents with cancer (project TREK): program feasibility and preliminary findings. J Pediatr Hematol Oncol. 2008;30:272-280.

36. van der Sluis IM, van den Heuvel-Eibrink MM. Osteoporosis in children with cancer. Pediatr Blood Cancer. 2008;50:474-478.

37. Tillmann V, Darlington AS, Eiser C, Bishop NJ, Davies HA. Male sex and low physical activity are associated with reduced spine bone mineral density in survivors of childhood acute lymphoblastic leukemia. J Bone Miner Res. 2002;17:1073-1080.

38. Zhang FF, Kelly MJ, Saltzman E, Must A, Roberts SB, Parsons SK. Obesity in pediatric ALL survivors: a meta-analysis. Pediatrics. 2014;133(3):e704-e715. 


\section{Publish your work in this journal}

The Open Access Journal of Clinical Trials is an international, peerreviewed, open access journal publishing original research, reports, editorials, reviews and commentaries on all aspects of clinical trial design, management, legal, ethical and regulatory issues, case record form design, data collection, quality assurance and data auditing

Submit your manuscript here: http://www.dovepress.com/open-access-journal-of-clinical-trials-journal methodologies. The manuscript management system is completely online and includes a very quick and fair peer-review system, which is all easy to use. Visit http://www.dovepress.com/testimonials.php to read real quotes from published authors. 